\title{
COBE starts its search for galactic fingerprints
}

\section{- Satellite launched after four-year delay - Looking for cosmic microwave irregularities}

\section{Washington}

THE launch on 18 November, of the COBE (Cosmic Background Explorer) satellite from Vandenberg Air Force Base in California marks the start of a longawaited astrophysical mission that had been delayed almost four years after the explosion of the space shuttle Challenger.

COBE's principal job is to look for irregularities in the cosmic microwave and far infrared backgrounds, an urgent task for cosmologists but one which has mostly passed beyond the capabilities of groundbased or balloon-flight experiments.

COBE was due to go on a shuttle flight soon after the Challenger disaster of January 1986, but NASA (National Aeronautics and Space Administration) decided shortly thereafter to switch it to a Delta rocket. This necessitated dismantling the finished satellite and putting the same instrumentation into a much smaller package. The reduction was achieved by omitting on-board thrusters, which would have been needed to propel COBE into its $900-\mathrm{km}$ high, approximately polar orbit from the low shuttle orbit. The Delta launcher can put $\mathrm{COBE}$ directly into place.

The \$160-million COBE satellite carries three instruments, and will scan the entire sky twice during it's one-year mission. By virtue of its polar orbit, COBE looks always at right angles to the direction of the Sun, whose light it must avoid to prevent damage to the detectors and interference with the measurements.

The two conical antennae of the Differential Microwave Radiometer will measure the difference in intensity of the cosmic microwave background, at three different frequencies, between two slightly displaced spots in the sky. This will yield an all-sky map of the variation of the microwave background from its average temperature of $2.7 \mathrm{~K}$.

Temperature variations at some level are predicted by all theories of galaxy formation, the primordial variations in the cosmological density which acted as the 'seeds' of modern galaxies must also have left their mark as small irregularities in the microwave background.

Over the past two decades, experiments done from the ground, balloons and shortflight rockets have produced no conclusive detection of temperature variations. When the earliest experiments were done, simple galaxy formation theories predicted temperature variations of as much as one part in a thousand, but as increasingly sophisticated instruments have brought the upper limit down to as little as a few parts in one hundred thousand, galaxy formation theories have been of necessity refined and adjusted to creep under the lowering wire of the measurements. There is a general feeling now that theory has few recourses left, and that if COBE does not find any temperature variations at a level perhaps an order magnitude less than the present best observations, then revision of standard cosmological ideas may be unavoidable.

The job of another of COBE's instruments, the Far Infrared Absolute Spectrophotometer, has become more crucial during the enforced delay of the satellite's launch. Last year, researchers from Nagoya University and the University of California at Berkeley published measurements obtained by a rocket-borne detector which indicated a substantial excess in the background intensity at wavelengths between 1.0 and $0.1 \mathrm{~mm}$, above the peak frequency of the microwave background, but below infrared emission from interstellar dust. The Nagoya-Berkeley team saw emission in this far infrared region an order of magnitude more intense than they expected; no convincing theoretical source has yet been identified, and some cosmologists believe that the detection is the result of some systematic instrumental error. COBE's far infrared detector should confirm or invalidate the Nagoya-Berkeley measurements, in either case telling cosmologists something about a region of the spectrum which, because of atmospheric opacity, has been little studied so far.

COBE's third detector, the Diffuse Infrared Background Experiment, goes to even longer wavelengths, and will look for emission which could be the accumulated radiation from distant, early galaxies.

By scanning the sky over a frequency range from microwave to infrared, $\mathrm{COBE}$ is in effect scanning the process of galaxy formation, from the imprint of density fluctuations retained by the microwave background to the faint, red-shifted emission reaching us now from the first generation of protogalaxies. Cosmologists are guaranteed to get something to think about, especially from the microwave experiment: if COBE finds the longsought irregularities, they will at last have some numbers to put into their theories; if it finds nothing, they will need some new theories.

\section{Nobel dispute continues}

\section{London}

DOMINIQUE Stéhelin, the French scientist who claims he should have shared this year's Nobel prize in physiology or medicine, has expounded his case in an open letter to the committee that recommended the winners, Michael Bishop and Harold Varmus. But the committee has no intention of responding.

In his letter, Stéhelin amplifies his view that it is unjust to have excluded from the prize the person who carried out the crucial experiments for the key discovery cited in the announcements of the awards. He argues that his exclusion is strongly detrimental because the "prize carries an unparalleled aura and prestige, lending it absolute and unquestioned authority in scientific quarters and the eyes of the public", and requests that the committee finds a way to repair the damage that has been done.

Apart from the fact that his name does not even appear in the documents that accompanied the announcement, most of Stéhelin's complaints are directed at comments made by one member of the committee in response to Stéhelin's initial outburst. Errling Norrby was quoted in newspapers as saying that the French researcher had not published anything of interest since 1976 , when the key paper by Stéhelin, Varmus, Bishop and Peter Vogt was published in Nature, and that whereas Bishop and Varmus's contributions had already been recognized by several prize awards, Stéhelin had none.

Stéhelin, who directs a unit within the Institut Pasteur in Lille, responds that among the more than 100 papers he has published since 1976 are reports of the discovery of six new oncogenes, and that he has been awarded four prizes, among which is the 1987 Jeantet prize for European biomedical research, which he shared with Sydney Brenner and Walter Gehring.

Jan Lindsten, secretary of the Nobel committee that recommended Varmus and Bishop, says that Norrby was speaking in a personal capacity, and that the committee will not be responding to Stéhelin. It is, he said, increasingly difficult to select the appropriate winners as research is increasingly a collaborative affair. While pointing out that the committee is empowered to consider only those who have been nominated, he refused to say whether there were any nominations for Stéhelin. Lindsten emphasized that the prize was given for more than the 1976 paper alone and that the parts played by Stéhelin and many others had been considered by the committee.

Peter Newmark 